\title{
Template Based Image Mosaics
}

\author{
Yoon-Seok Choi, Bon-Ki Koo, and Ji-Hyung Lee \\ CG Research Team, Digital Content Research Division, \\ Electronics and Telecommunications Research Institute, \\ 161 Gajeong-dong, Yuseong-gu, Daejeon, 305-350, Replublic of korea \\ \{ys-choi, bkkoo, ijihyung\}@etri.re.kr
}

\begin{abstract}
We propose an image mosaic method based on tile template. Compared with a conventional mosaic which is comprised of the circle or the square tiles, our approach uses the arbitrary shaped tiles. But an arbitrary shaped tile leaves the excessive gap between tiles in the mosaic construction. Then the gap makes it difficult to preserve the fine detail of an original image. Our approach controls overlap between tiles to minimize the gap by using the mask technique and adopts stackable technique to represent the fine detail of a source image automatically.
\end{abstract}

Keywords: Non Photorealistic Rendering, Mosaic Rendering, Image Mosaic.

\section{Introduction}

Non photorealistic rendering is the general term for rendering techniques that use various painting materials such as pen, oil, watercolor, and so on. Several studies have been made on the simulated mosaic in the field of non photorealistic rendering. Mosaic is an art technique which produces an art work by assembling the pattern or the form of a tile, a marble, the paper, and the flat glass to the plane canvas.

This paper shows a mosaic rendering method using the various shaped tiles including circle or rectangle tiles.

\section{Previous Work}

In mosaic rendering, several research were proposed including the colored paper mosaics [1, photomosaics [2, the square tile [3, and so on. According to the method for determining the shape, the color, the location, and the orientation of a tile, mosaic rendering produces the various images.

Seo 1 proposed mosaic effects of color-paper. Seo could express a hand-made mosaic effect by using the texture as well as the torn-paper effect. Photomosaics 2] made mosaics from photographs instead of solid colored tiles and arranged small images in a rectangular grid. From a distance, small images are seen together as a large image. Hausner [3] decided the location of square tiles by using the centroidal voronoi diagram and varied the orientations of the tiles 
using the direction field. He generated random square tiles on the image plane and then rearranged them iteratively until the area of tiles is maximized to preserve input image edges. Dobashi et al. 4. tried to improve Hausner's approach by using the different tile shapes. Park [5] suggested an algorithm using multilayered photomosaic with stackable tiles which are independent, rotatable and non-rectangular in shape, such as coins, dishes, and so on.

A template refers to the part in which the tile image shows up in a screen. The shapes of templates might be more free than a circle or a square. We explain our approach which generates mosaic image based on a template of a tile with mask control and stackable tiles.

\section{Template Based Image Mosaics}

To produce mosaic, we use three types of masks: edge, energy, and write masks. The edge and energy masks are necessary for preserving the edge of an original image and representing a beauty of margin respectively. The energy mask is generated based on the light intensity value of image. The edge mask includes edge information generated by edge detection algorithm. When rendering process positions a tile in a segment, which is the divided area of an image, the write mask informs whether the segment is vacant or not. If it is vacant the mask stores the information which target image is filled with the tile. The edge and energy masks operate analogously to the write mask. The mosaic production has two steps. In the first step, the empty space of an image is filled with a tile. In the second step, the expression of an image is improved by piling up a tile with a multi-layer. Fig 1 shows the process to produce a mosaic image.

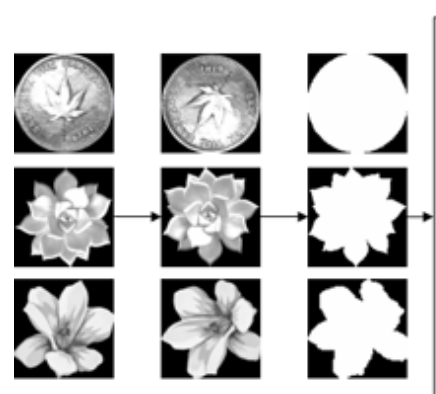

(a) (b)

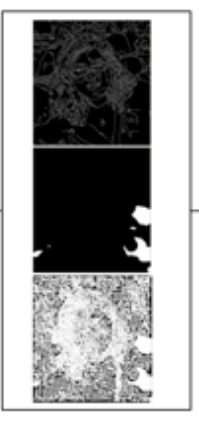

(d)

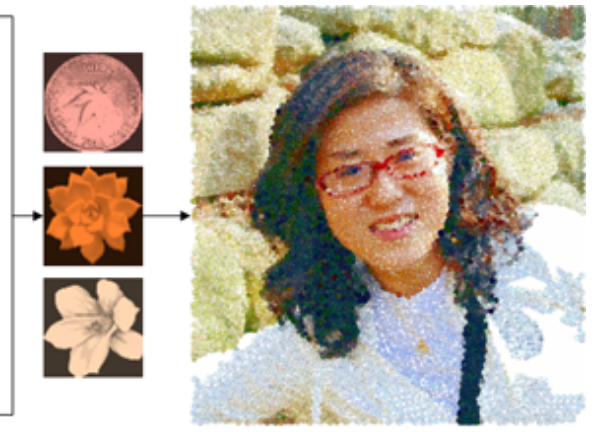

(e)

(f)

Fig. 1. Process diagram (a) various shaped tiles (b) rotated tiles (c) templates of tiles (d) edge, energy, and write masks (e) colored tiles (f) final image

\subsection{Tile Alignment}

First of all, the write mask with the the equal size to that of the target mosaic is set up as 0 . To generate multi-angled tiles, we use flood filling method. The flood 
filling method searched repeatedly space and decided a good position to locate a tile while rotating a tile with comparing a template of a tile with three types of masks. Whenever a space is filled with tiles, the space of write mask which is corresponding to the filled space is set to 1 . In order to minimize gap between tiles, the size of a tile can be reduced. The positions of tiles are determined through a comparison between the template extracted from the tiles and the masks. The filling process is repeated until there is no space for tile filling.

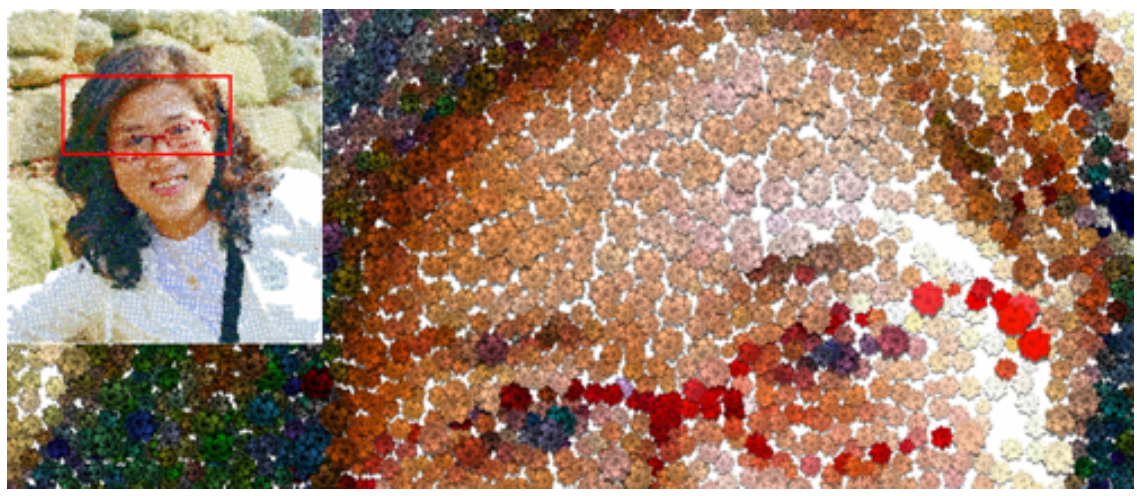

Fig. 2. Fine details using stackable tiles

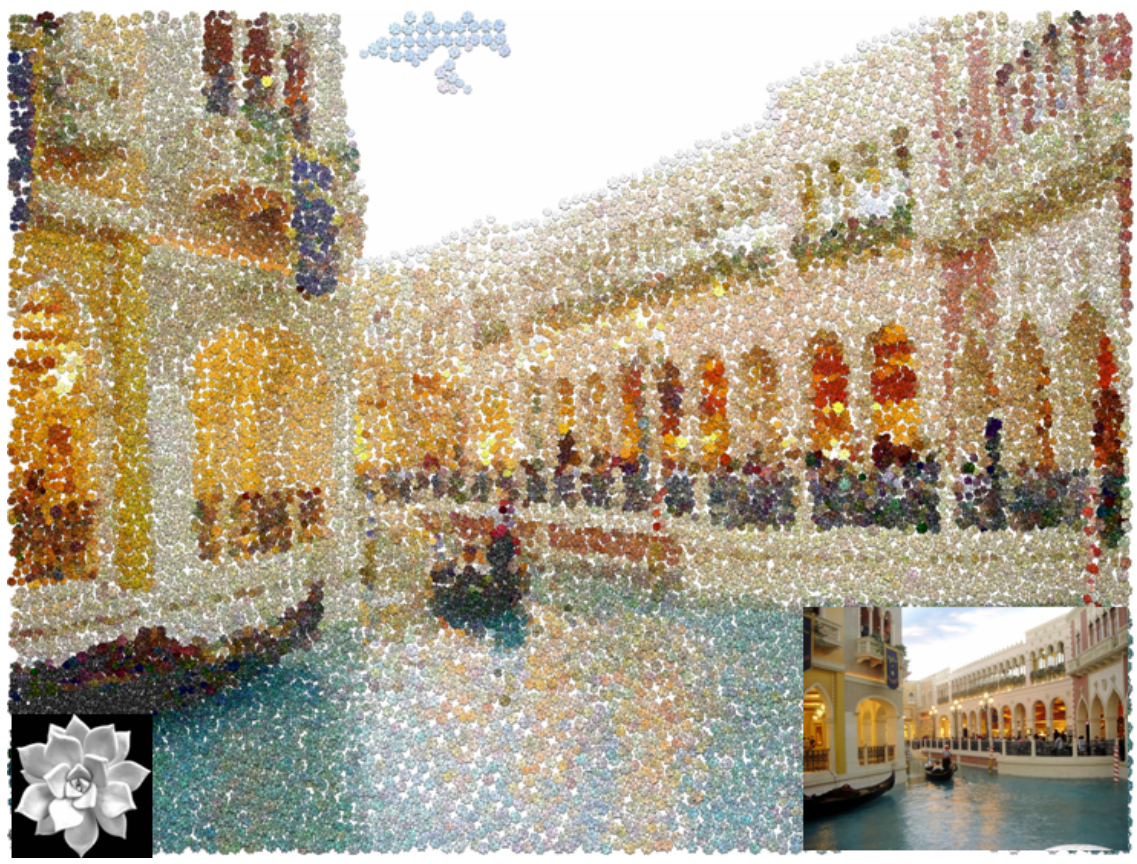

Fig. 3. Template based Mosaics Rendering: Source image, tile, and the result 


\subsection{Detail Enhancement with Stackable Tiles}

We applied a conventional photomosaic algorithm and merged it with a shadow effect as Park's approach [5]. Comparing original image with a colored tile with the RGB value, we decided the inappropriate position of each tile. In this case, the edge mask which is used in the previous step was not used. For improving the fine details of mosaic image, stackable tiles are used (Fig. 2).

\section{Conclusion and Future Work}

In this paper, we tried to generate mosaic image based on a template of tile using mask control and stackable tiles. The three types of masks are produced based on the source image and tiles are aligned through comparison with the masks. These masks control not only the positions of the tiles but also the sizes of them. Finally in order to enhance images expression we adopted stackable tiles. However, as to the overlay control through the mask control, the gap minimization between tiles is limited. Two dimension nesting problem is from now on applied for resolving the gap minimization problem between the tiles.

\section{Acknowledgement}

This work was supported by the IT R\&D program of MIC/IITA. [2005-S082-02, Development of Non-Photorealistic Animation Technology]

\section{References}

1. Seo, S., Park, Y., Kim, S., Yoon, K.: Colored Paper Mosaic Rendering. In: SIGGRAPH '01: Proceedings of Conference on Sketches and Abstaracts and Applications, p. 157 (2001)

2. Silverts, R., Hawley, M.: Photomosaics. Henry Holt, New York (1997)

3. Alejo, H.: Simulating Decorative Mosaics. In: SIGGRAPH '01: Proceedings of the 28th annual conference on Computer graphics and interactive techniques, pp. 573580 (2001)

4. Yoshinori, D., Toshiyuki, H., Henry, J., Tomoyuki, N.: A method for creating mosaic images using voronoi diagrams. In: EUROGRAPHICS '02: Proceedings Annual Conference of the European Association for Computer Graphics (2002)

5. Park, J.: The Coinage Project \#1: "Time Is Money". Leonardo 38(2), 124 (2005) 